\title{
Carboxypeptidase M: a biomarker for the discrimination of well-differentiated liposarcoma from lipoma
}

\author{
Michele R Erickson-Johnson, Amber R Seys, Christopher W Roth, Allison A King, \\ Rachael L Hulshizer, Xiaoke Wang, Yan W Asmann, Ricardo V Lloyd, Eapen K Jacob \\ and Andre M Oliveira
}

Department of Laboratory Medicine and Pathology, Mayo Clinic, Rochester, MN, USA

\begin{abstract}
The discrimination between well-differentiated liposarcomas/atypical lipomatous tumors and lipomas can be diagnostically challenging at the histological level. However, cytogenetic identification of ring and giant rod chromosomes supports the diagnosis of well-differentiated liposarcoma/atypical lipomatous tumor. These abnormal chromosomes are mainly composed of amplified genomic sequences derived from chromosome 12q13-15, and contain several genes, including MDM2, CDK4 (SAS), TSPAN31, HMGA2, and others. MDM2 is consistently amplified in well-differentiated liposarcomas/atypical lipomatous tumors, and up to $25 \%$ in other sarcomas. As part of a large genomic study of lipomatous neoplasms, we initially found CPM to be consistently amplified in well-differentiated liposarcomas/atypical lipomatous tumors. To further explore this initial finding, we investigated the copy number status of MDM2 and CPM by fluorescent in situ hybridization (FISH) on a series of 138 tumors and 17 normal tissues, including 32 well-differentiated liposarcoma/atypical lipomatous tumors, 63 lipomas, 11 pleomorphic lipomas, 2 lipoblastomas, 30 other tumors and 17 normal fat samples. All 32 well-differentiated liposarcoma/atypical lipomatous tumors showed amplification of MDM2 and CPM, usually $>20$ copies per cell. The other tumors lacked MDM2 and/or CPM amplification. Chromogenic in situ hybridization confirmed the above results on a subset of these tumors $(n=27)$. These findings suggest that identification of CPM amplification could be used as an alternative diagnostic tool for the diagnosis of welldifferentiated liposarcoma/atypical lipomatous tumors.

Modern Pathology (2009) 22, 1541-1547; doi:10.1038/modpathol.2009.149; published online 9 October 2009
\end{abstract}

Keywords: lipoma; well-differentiated liposarcoma; CPM; MDM2; FISH

Well-differentiated liposarcomas/atypical lipomatous tumors are among the most common sarcomas presenting in older adults. ${ }^{1-4,8}$ These tumors often arise in deep tissues as slow growing masses and can occasionally be difficult to distinguish morphologically from lipomas. ${ }^{1-4,8}$ Cytogenetically, welldifferentiated liposarcomas/atypical lipomatous tumors are characterized by supernumerary ring and large marker chromosomes. These abnormal chromosomes are mainly composed of amplified genomic sequences derived from chromosome bands 12q13-15, and contain several genes. Among them, MDM2 seems to be the most consistently

Correspondence: Dr AM Oliveira, MD, Department of Laboratory Medicine and Pathology, Mayo Clinic, Hilton 11th, 200 First Street SW, Rochester, MN 55905, USA.

E-mail:oliveira.andre@mayo.edu

Received 19 July 2009; revised 2 September 2009; accepted 3 September 2009; published online 9 October 2009 amplified gene. ${ }^{1-4}$ During a high-resolution microarray comparative genomic hybridization experiment on a series of lipomatous neoplasms, we discovered that CPM, a gene that encodes carboxypeptidase $M$, was consistently amplified in liposarcomas but not in different subtypes of lipoma or normal fat.

In this study, we compared $M D M 2$ and $C P M$ on a series of lipomatous tumors to establish whether $C P M$ could be used clinically to discriminate well-differentiated liposarcoma/atypical lipomatous tumors from lipomas.

\section{Materials and methods}

Tumor Samples

After receiving Institutional Review Board approval, formalin-fixed and paraffin-embedded specimens were identified from the archives of the Department 
Table 1 Results for $M D M 2$ and $C P M$

\begin{tabular}{|c|c|c|c|}
\hline Soft tissue tumor & MDM2 & $C P M$ & Interpretation \\
\hline Well-differentiated liposarcoma/atypical lipomatous tumor $(n=17)$ & $17 / 17$ & $17 / 17$ & Amplification $^{\mathrm{a}}$ \\
\hline Lipoma $(n=27)$ & $0 / 27$ & $0 / 27$ & Negative \\
\hline Pleomorphic sarcoma $(n=4)$ & $0 / 4$ & $0 / 4$ & Aneuploidy ${ }^{\mathrm{b}}(4 / 4)$ \\
\hline Pleomorphic lipoma $(n=4)$ & $0 / 4$ & $0 / 4$ & Aneuploidy ${ }^{\mathrm{b}}(4 / 4)$ \\
\hline Angiomyofibroblastoma $(n=1)$ & $0 / 1$ & $0 / 1$ & Negative \\
\hline Myxoid liposarcoma $(n=8)$ & $0 / 8$ & $0 / 8$ & Aneuploidy (2/8) \\
\hline Sarcomatoid renal cell carcinoma $(n=2)$ & $0 / 2$ & $0 / 2$ & Negative \\
\hline Pleomorphic liposarcoma $(n=1)$ & $0 / 1$ & $0 / 1$ & Negative \\
\hline
\end{tabular}

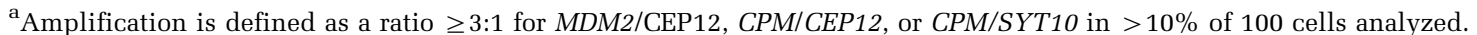

${ }^{\mathrm{b}}$ Aneuploidy is defined as a chromosome number that are not an exact multiple of the haploid set of chromosomes.

of Laboratory Medicine and Pathology at the Mayo Clinic, Rochester, MN, USA. Hematoxylin and eosin (H\&E)-stained sections were reviewed in all cases for diagnostic confirmation. The verified diagnostic categories included well-differentiated liposarcoma/ atypical lipomatous tumors $(n=32)$, ordinary lipomas $(n=63)$, myxoid liposarcomas $(n=7)$, undifferentiated pleomorphic sarcomas $(n=15)$, pleomorphic lipomas $(n=11)$, sarcomatoic renal cell carcinomas $(n=2)$, angiomyofibroblastoma $(n=2)$, low-grade myxofibrosarcoma $(n=2)$, lipoblastoma $(n=2)$, hibernoma $(n=1)$, nodular fasciitis $(n=1)$, and normal fat samples $(n=17)$ (Tables 1 and 2).

\section{FISH}

All cases were evaluated with laboratory-developed fluorescent in situ hybridization (FISH) probe sets. Bacterial artificial chromosome (BAC) clones spanning the $C P M$ and $M D M 2$ loci, 12q13-15, were obtained from the Children's Hospital Oakland Research Institute (Oakland, CA, USA). BAC clones for $C P M$ are RP11-717F7, RP11-426B12, RP11630N19, RP11-1104N20, and RP11-1036O8. BAC clones for MDM2 are RP11-61F20, RP11-816C9, RP11-185H13, RP11-450G15. BAC clones spanned the $C P M$ and $M D M 2$ genes and are $729 \mathrm{~kb}$ and $765 \mathrm{~kb}$, respectively. The chromosome 12 reference probes for FISH and chromogenic in situ hybridization (CISH) were CEP12 (Abbott Laboratory, North Chicago, IL, USA) and the chromosome 12 pericentromeric gene SYT10 (RP11-8P13, RP11-267D19, RP11-855O3, RP11-88P4, RP11-102G23, RP1151012 and RP11-1143D16), respectively. All of the identities for the BAC clones were individually confirmed by PCR and by hybridization on metaphase preparations from the peripheral blood of five normal individuals. Analytical sensitivity and specificity of each probe were calculated and their performance on paraffin-embedded tissue was verified on numerous normal tissue types, including the skeletal muscle, adipose tissue, and others. Amplification was defined as CPM/CEP12 and/or $M D M 2 / C E P 12$ or $C P M / S Y T 10$ ratio $\geq 3$. Normal
Table 2 Results for $C P M$ only (independent set)

\begin{tabular}{lll}
\hline Soft tissue tumor & CPM & Interpretation \\
\hline $\begin{array}{l}\text { Well-differentiated liposarcoma/ } \\
\text { atypical lipomatous tumor }(n=15)\end{array}$ & $15 / 15$ & Amplification \\
Lipoma $(n=36)$ & $0 / 36$ & Negative \\
Pleomorphic sarcoma $(n=11)$ & $0 / 11$ & Aneuploidy $(11 / 11)$ \\
Pleomorphic lipoma $(n=7)$ & $0 / 7$ & Aneuploidy $(7 / 7)$ \\
Angiomyofibroblastoma $(n=1)$ & $0 / 1$ & Negative \\
Myxoid liposarcoma $(n=1)$ & $0 / 1$ & Negative \\
Hibernoma $(n=1)$ & $0 / 1$ & Negative \\
Nodular fasciitis $(n=1)$ & $0 / 1$ & Negative \\
Low-grade myxofibrosarcoma $(n=2)$ & $0 / 2$ & Negative \\
\hline
\end{tabular}

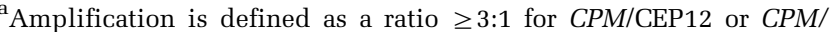
SYT10 in $>10 \%$ of 100 cells analyzed.

${ }^{\mathrm{b}}$ Aneuploidy is defined as a chromosome number that are not an exact multiple of the haploid set of chromosomes.

structures that could be readily identified under the 4', 6-diamidino-2-phenylindole (DAPI) staining on thin sections, such as the blood vessels and epidermis, were used as internal controls for the cases analyzed. Normal signal patterns were established by scoring 100 cells from each normal tissue.

DNA isolation was carried out using the Qiagen Plasmid Maxi kit (Qiagen, Valencia, CA, USA). DNA was labeled using a nick translation kit (Abbott Laboratory). Interphase molecular cytogenetic studies were carried out using $4-\mu \mathrm{m}$ paraffin-embedded thin sections that were deparaffinized twice in xylene (15 min pretreatment), dehydrated once in $100 \%$ ethanol $(5 \mathrm{~min})$, and treated with $10 \mathrm{mmol} / \mathrm{l}$ citric acid (10 min, in a humidified microwave). Tissue sections were incubated in warm $\left(37^{\circ} \mathrm{C}\right)$ sodium chloride-sodium citrate buffer $(2 \times$ SSC $)$ for $5 \mathrm{~min}$. Protein was digested with Digest-All 3 (Invitrogen Corporation, Carlsbad, CA, USA). After a brief wash in phosphate-buffered saline $(1 \times$ PBS), slides were sequentially dehydrated in ethanol (70, 80 , and $100 \%$ ) and air dried at room temperature. Tissue sections were denatured at $85^{\circ} \mathrm{C}$ for $5 \mathrm{~min}$, and BAC probe hybridization was performed overnight in a humidified chamber at $37^{\circ} \mathrm{C}$. Tissue sections were washed in $0.1 \%$ Nonidet P-40 (NP40) 

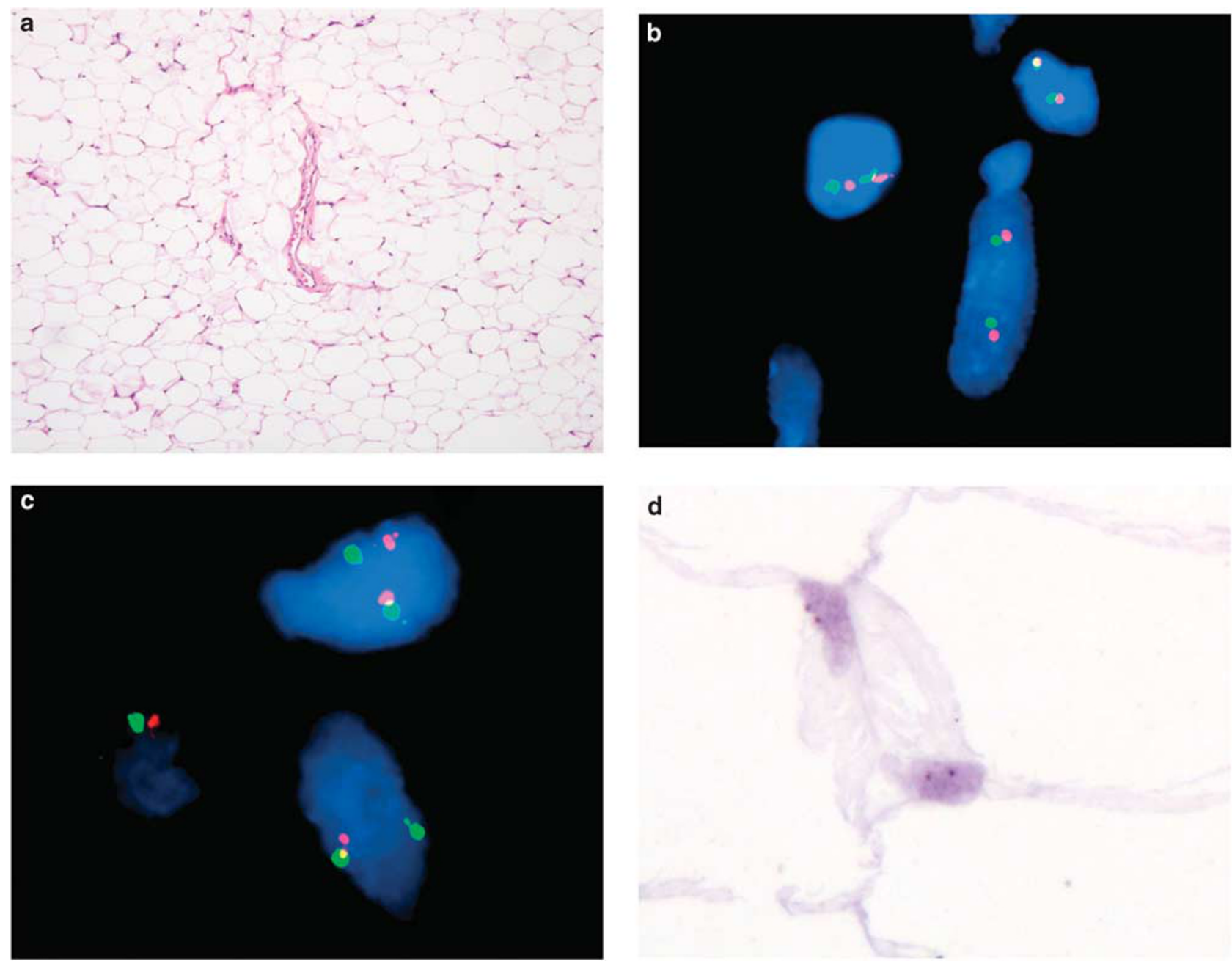

Figure 1 Lipoma. (a) Histological features of a representative case. (b) and (c) FISH images of this case with MDM2 and CPM probes, respectively. Orange signals represent either MDM2 or CPM. Green signals show chromosome 12 centromeres. (d) Normal CISH pattern for $C P M$ in the same case. Brown signals represent normal copies of $C P M$.

in $2 \times \mathrm{SSC}$ at $76^{\circ} \mathrm{C}$ for $2 \mathrm{~min}$ and then washed in the same solution at room temperature for 1 min. Slides were mounted in Vectashield mounting medium (Vector Laboratories, Burlingame, CA, USA) with $1.5 \mu \mathrm{g} / \mathrm{ml}$ DAPI medium (Vector Laboratories). Tumor samples were considered positive for amplification if a ratio $\geq 3: 1 \mathrm{CPM} / \mathrm{CEP} 12$ and/or $M D M 2 /$ CEP12 was found in $>10 \%$ of 100 cells. Tumors were evaluated and scored by three independent investigators (MEJ, ARS, and CWR).

\section{CISH}

Using the same DNA extracted for FISH probes ( $C P M, M D M 2$, and SYT10), DNA was labeled using the DIG DNA-labeling mix (Roche Diagnostics, Mannheim, Germany). In brief, $5 \mu \mathrm{l}$ of $0.2 \mu \mathrm{g} / \mathrm{ml}$ DNA and $2.5 \times$ random primers were mixed and placed on a thermal cycler at $99^{\circ} \mathrm{C}$ for $5 \mathrm{~min}$, followed by placing on ice for $1 \mathrm{~min}$. In all, $5 \mu \mathrm{l} 10 \times \mathrm{DIG}$-dNTP
(Roche Diagnostics), $19 \mu \mathrm{l}$ water, and $1 \mu \mathrm{l}$ Klenow fragment (Roche Diagnostics) were added, gently mixed, and placed on a thermal cycler overnight at $37^{\circ} \mathrm{C}$. DNA was then purified as per the manufacturer's instructions using a microspin s-200HR column (GE Healthcare, Fairfield CT, USA) and precipitated by the addition of $1 \mu \mathrm{l}$ of $20 \mu \mathrm{g} / \mathrm{ml}$ glycogen (Invitrogen Corporation) and $33 \times 7.5 \mathrm{M}$ ammonium acetate (Sigma-Aldrich, St Louis, MO, USA) and $2 \times 100 \%$ ethanol. Samples were precipitated overnight in an ethanol bath at $-80^{\circ} \mathrm{C}$. Labeled probe was centrifuged at 14000 r.p.m. for $15 \mathrm{~min}$ at $4^{\circ} \mathrm{C}$ and pellets were resuspended in $100 \mu \mathrm{l}$ LSI/WCP hybridization buffer (Abbott Laboratory). CISH slides were prepared following the FISH processing procedure until the addition of probe. CISH probe $(5 \mu \mathrm{l})$ and $2 \mu \mathrm{l}$ COT-1 (Invitrogen Corporation) were added to the slides and hybridized at $80^{\circ} \mathrm{C}$ for $5 \mathrm{~min}$, and then held at $37^{\circ} \mathrm{C}$ overnight. On the following day, coverslips were removed and slides were washed in $76^{\circ} \mathrm{C} 0.5 \times \mathrm{SSC}$ for $2 \mathrm{~min}$, 

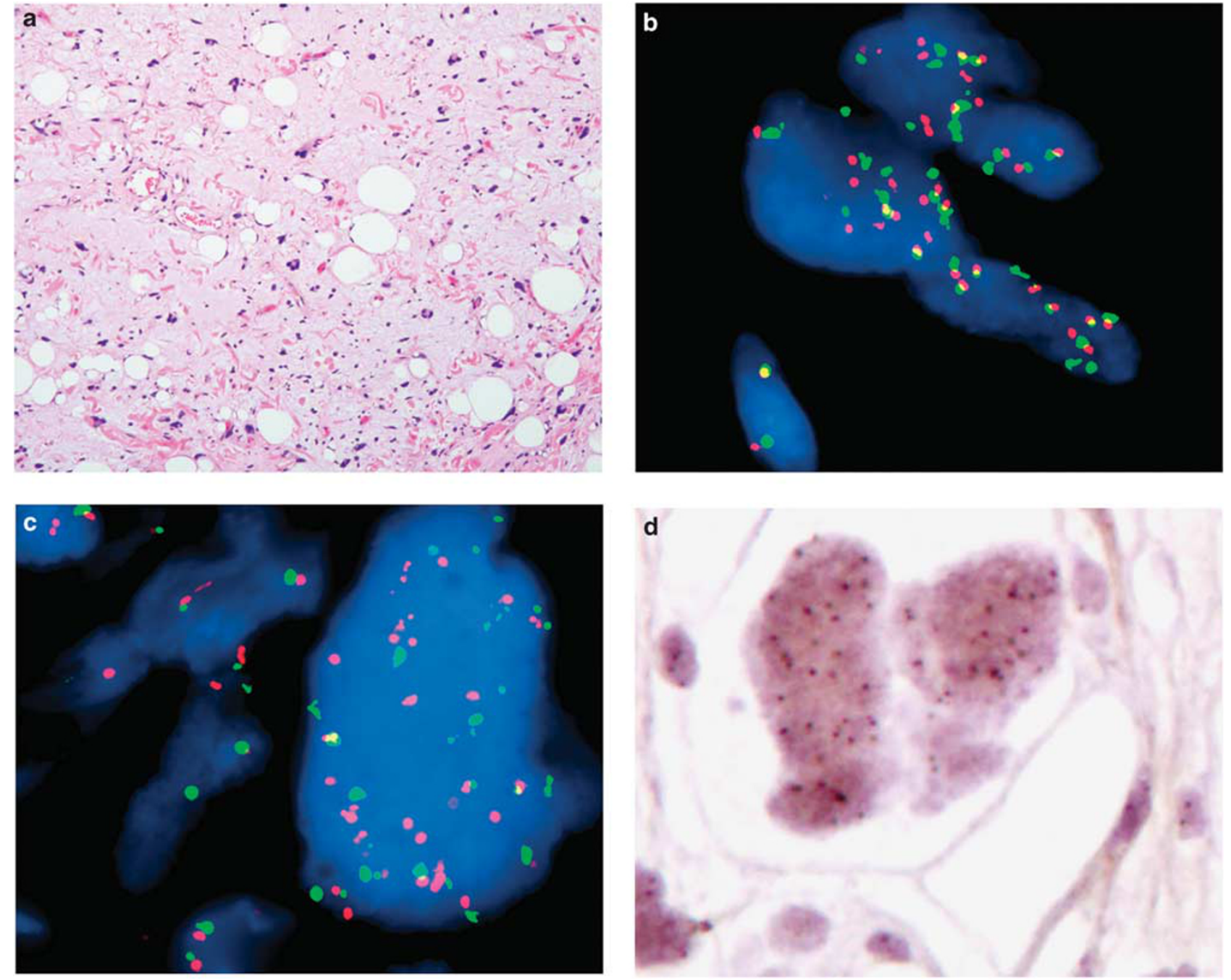

Figure 2 Pleomorphic lipoma. (a) Histological features of a representative case. (b) and (c) FISH images of this case with $M D M 2$ and $C P M$ probes, respectively. Orange signals represent either MDM2 or CPM. Green signals show chromosome 12 centromeres. (d) CISH image of a $C P M$ probe hybridized to a pleomorphic lipoma. Scattered brown signals represent multiple copies of $C P M$ without amplification (clustering). These images show FISH and CISH signatures consistent with numerical abnormalities of chromosome 12 but no amplification of either MDM2 or CPM (CPM/SYT10 and MDM2/CEP12 ratios=1).

followed by a wash in $1 \times$ PBS for 2 min. Slides were visualized by using the Spot-Light CISH Polymer detection kit (Zymed Laboratories, South San Francisco, CA, USA). Briefly, slides were incubated in $3 \%$ hydrogen peroxide for $5 \mathrm{~min}$ followed by three washes in PBS for 2 min each. Slides were blocked using in CAS-Block (Invitrogen Corporation) for $10 \mathrm{~min}$. Reagent B (mouse-anti DIG) was added and incubated for $60 \mathrm{~min}$ followed by three washes in $1 \times$ PBS, followed by the addition of 3'3-diaminobenzidine (DAB), incubated for $5 \mathrm{~min}$, and washed in PBS. Following the addition of DAB, the slides were counterstained with hematoxylin, dehydrated in an ethanol series (70, 85, and 100\%), immersed in xylene, and coverslipped using histomount. Tumor samples were considered positive for amplification if a ratio $\geq 3: 1 C P M / S Y T 10$ or MDM2/ $S Y T 10$ was found in $>10 \%$ of 100 cells analyzed.
Tumors were evaluated and scored by two independent investigators (MEJ and AMO).

\section{Comparative Genomic Hybridization}

This study is part of a major genomic analysis of a large series of lipomatous neoplasms, which is currently in progress. Briefly, $C P M$ was discovered using the Human CNV370-Quad BeadChip (Illumina, San Diego, CA, USA), which contains over 370000 markers. Analysis was by multiple copy number variation (CNV) detection tools, including cnvPartition (Illumina), CGHAnalyzer (open source software from University of Pennsylvania), and Segmentation algorithm in Partek Genomic Suit (Partek Incorporated, St Louis, MO, USA). 

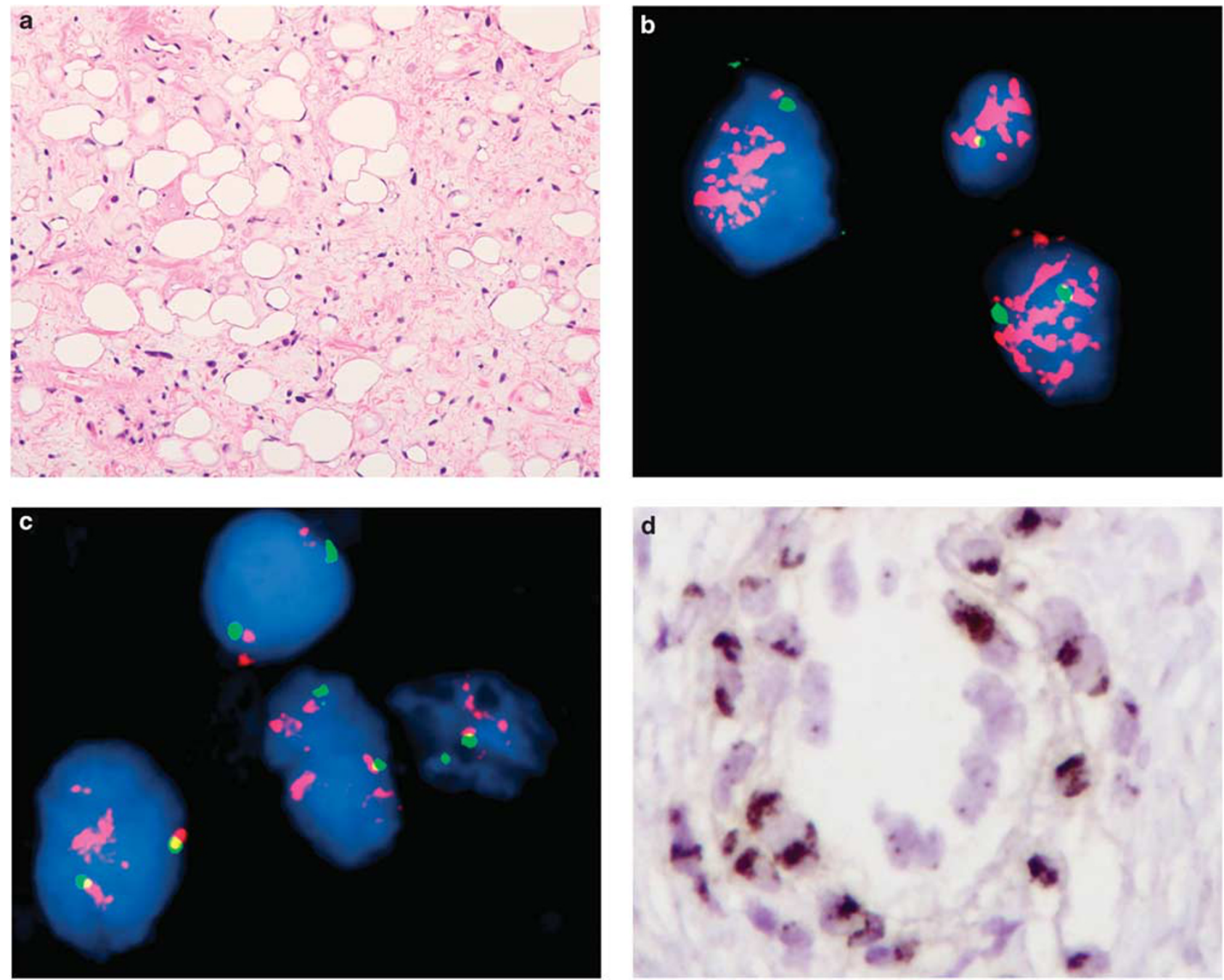

Figure 3 Well-differentiated liposarcoma/atypical lipomatous tumor. (a) Histological features of a representative case. (b) and (c) FISH images of this case show MDM2 and CPM amplification, respectively. Orange signals represent either MDM2 or CPM. Green signals show chromosome 12 centromeres. (d) CISH image of CPM probe hybridized to this case; clusters of brown signals represent CPM amplification. These images show FISH and CISH signatures consistent with the amplification of these genes (CPM/SYT10 and MDM2/ CEP12 ratios $\geq 3$ )

\section{Results}

\section{FISH and CISH}

All cases were found to have detectable and analyzable signals. The data in Tables 1 and 2 depict the results for each tumor category. All 32 welldifferentiated liposarcomas/atypical lipomatous tumors were found to have amplification of MDM2 and/or CPM, usually with $>20$ copies per cell (Tables 1 and 2, and Figure 3). Lipomas, lipoblastomas, and normal adipose tissue samples showed a normal FISH signal pattern with two copies of MDM2 and CPM (Tables 1 and 2, and Figure 1). Pleomorphic lipomas and pleomorphic liposarcomas showed FISH signal patterns consistent with numerical abnormalities of chromosome 12 but without amplification of either $C P M$ or $M D M 2$ (Figure 2). Two myxoid liposarcomas (29\%) exhibited patterns consistent with monosomy 12 or loss of the $C P M / M D M 2$ loci (Tables 1 and 2). The remaining cases showed normal FISH signal patterns. Using the same FISH scoring criteria, all 27 cases analyzed by CISH (21 lipomas, 4 liposarcomas, and 2 normal fat samples) were found to have detectable and analyzable signals. CISH produced results similar to FISH, resulting in normal patterns for lipomas and normal fat samples (Figure 1), and amplification for liposarcoma (Figure 3). Pleomorphic lipomas and myxoid liposarcomas showed signal patterns consistent with numerical chromosomal abnormalities of chromosome 12 without amplification, similar to what was seen with FISH (Figure 2).

\section{Discussion}

Well-differentiated liposarcomas/atypical lipomatous tumors with minimal or no cytological atypia 
can be difficult to distinguish from lipomas and fibrolipomas..$^{1,3,5-9}$ However, the identification of ring and giant rod chromosomes by cytogenetic analysis supports the diagnosis of well-differentiated liposarcomas/atypical lipomatous tumor. These abnormal chromosomes are mainly composed of amplified genomic sequences derived from chromosome bands 12q13-15 and contain several genes, including MDM2, CDK4 (SAS), TSPAN31, HMGA2, among others. ${ }^{8-21}$ MDM2 seems to be amplified in almost all cases of well-differentiated liposarcoma/atypical lipomatous tumors, especially when molecular cytogenetic analysis is carried out. ${ }^{1,9}$ In addition, MDM2 amplification has been described in up to $25 \%$ of other sarcomas. ${ }^{1-3}$

As a part of large genomic study of lipomatous neoplasms, we found $C P M$ to be consistently amplified well-differentiated liposarcomas/atypical lipomatous tumors in a CNV screening array CGH. ${ }^{22}$ Our experiments showed co-amplification of $M D M 2$ and $C P M$ in all well-differentiated liposarcoma/ atypical lipomatous tumors evaluated in an initial test set. An independent group of well-differentiated liposarcoma/atypical lipomatous tumors was then tested only for CPM. Amplification of this gene was observed in all instances. All other tumors were negative for $C P M$ amplification. These results correlate with previous studies that showed consistent amplification of MDM2 in well-differentiated liposarcoma/atypical lipomatous tumors, but not in lipomas. ${ }^{1,9,22}$ FISH results were also corroborated by a CISH assay in 27 of these tumors. Both FISH and CISH are closely related techniques resulting in the ability to enumerate chromosome and/or gene status. FISH requires a fluorescent microscope, trained technologists, and the signals of slides fade over time. In the other hand, CISH signals can be read out by any pathologist in a bright field microscope, and the findings can be directly correlated with specific morphological details of the tumor. However, CISH signals may be more difficult to be visualized and individually enumerated, especially if one is dealing with low levels of gene amplification. The final decision on which technique to use will depend on the comfort level and familiarity of the performing laboratory with each one of them.

CPM spans $\sim 113 \mathrm{~kb}$ of genomic DNA, contains 11 exons, and encodes for a membrane-bound zincdependent protease that cleaves C-terminal basic residues from peptides and proteins. CPM is located $11 \mathrm{~kb}$ downstream from MDM2 and the encoded protein has been implicated in many functions, such as adipose tissue differentiation, osteogenesis, inflammation, and coagulation. ${ }^{23} C P M$ is expressed at high levels in many tissues and cell types, including the pulmonary membranes, placenta, and kidney. ${ }^{23}$ Gene expression profiling has shown $C P M$ transcriptional upregulation in response to specific external stimuli, which may be used as an indicator of body's response to infection or disease. $^{23}$ Recently, $C P M$ has been found to be upregulated in soft tissue sarcomas, such as synovial sarcoma, gastrointestinal stromal tumors, and dedifferentiated/pleomorphic liposarcomas. ${ }^{23}$ However, whether $C P M$ has an important oncogenic role in those tumors or in liposarcomas is still unknown.

In summary, we have found that CPM is consistently amplified in well-differentiated liposarcoma/ atypical lipomatous tumors but not in ordinary or pleomorphic lipomas. These results offer another avenue for the investigation of the biology of welldifferentiated liposarcomas and suggest that $C P M$ could be used as an alternative and novel diagnostic tool for these tumors.

\section{Acknowledgement}

This study was in part supported by the Fraternal Order of Eagles Foundation Award 237 and Mayo Clinic CR20 Award 14546.

\section{Disclosure/conflict of interest}

The authors declare no conflict of interest.

\section{References}

1 Jacob EK, Erickson-Johnson MR, Wang X, et al. Assessment of MDM2 amplification using fluorescence in situ hybridization of paraffin embedded tissues discriminates atypical lipomatous tumors from lipomas. Mod Pathol 2006;19(13A):45.

2 Sirvent N, Forus A, Lescaut W, et al. Characterization of centromere alterations in liposarcomas. Genes Chromosomes Cancer 2000;29:117-129.

3 Mef Nilbert AR, Felix M, Paul SM, et al. Characterization of the 12q13-15 amplicon in soft tissue tumors. Cancer Genet Cytogenet 1995;83:32-36.

4 Nilsson M, Meza-Zepeda LA, Mertens F, et al. Amplification of chromosome 1 sequences in lipomatous tumors and other sarcomas. Int J Cancer 2004; 109:363-369.

5 Skubitz K, Cheng E, Clohisy D, et al. Differential gene expression in liposarcoma, lipoma, and adipose tissue. Cancer Invest 2005;23:105-118.

6 Pilotti S, Della Torre G, Lavarino C, et al. Distinct mdm2/p53 expression patterns in liposarcoma subgroups: implications for different pathogenetic mechanisms. J Pathol 1997;181:14-24.

7 Weiss SW. Enzinger and Weiss's Soft Tissue Tumors 4th edn. Mosby: St Louis, MO, 2001.

8 Weaver J, Downs-Kelly E, Goldblum JR, et al. Fluorescence in situ hybridization for MDM2 gene amplification as a diagnostic tool in lipomatous neoplasms. Mod Pathol 2008;21:943-949.

9 Trahan S, Erickson-Johnson MR, Rodriguez F, et al. Formation of the 12q14-q15 amplicon precedes the development of a well-differentiated liposarcoma arising from a nonchondroid pulmonary hamartoma. Am J Surg Pathol 2006;30:1326-1329. 
10 Fletcher C, Unni K, Mertens F, (eds) WHO Classification of Tumours; Pathology and Genetics: Tumours of Soft Tissue and Bone. IARC Press: Lyon, France, 2002.

11 Laurino L, Furlanetto A, Orvieto E, et al. Welldifferentiated liposarcoma (atypical lipomatous tumors). Semin Diagn Pathol 2001;18:258-262.

12 Pedeutour F, Forus A, Coindre J, et al. Structure of the supernumerary ring and giant rod chromosomes in adipose tissue tumors. Genes Chromosomes Cancer 1999;24:30-41.

13 Gisselsson D, Hoglund M, Mertens F, et al. The structure and dynamics of ring chromosomes in human neoplastic and non-neoplastic cells. Hum Genet 1999; 104:315-325.

14 Borden E, Baker L, Bell R, et al. Soft tissue sarcomas of adults: state of the translational science. Clin Cancer Res 2003;9:1941-1956.

15 Berner J, Forus A, Elkahloun A, et al. Separate amplified regions encompassing CDK4 and MDM2 in human sarcomas. Genes Chromosomes Cancer 1996;17:254-259.

16 Ida CM WX, Erickson-Johnson MR, Wenger DE, et al. Primary retroperitoneal lipoma: a soft tissue pathology heresy?: report of a case with classic histologic, cytogenetics, and molecular genetic features. Am J Surg Pathol 2008;32:951-954.
17 Gustafson P, Akerman M, Alvegard T, et al. Prognostic information in soft tissue sarcoma using tumour size, vascular invasion and microscopic tumour necrosisthe SIN-system. Eur J Cancer 2003;39:1568-1576.

18 Nielsen T, West R, Linn S, et al. Molecular characterization of soft tissue tumours: a gene expression study. Lancet 2002;359:1301-1307.

19 Golub T, Slonim D, Tamayo P, et al. Molecular classification of cancer: class discovery and class prediction by gene expression monitoring. Science 1999;286:531-537.

20 Helman L, Meltzer P. Mechanisms of sarcoma development. Nat Rev Cancer 2003;3:685-694.

21 Fanburg-Smith JC, Miettinen M. Liposarcoma with meningothelial-like whorls: a study of 17 cases of a distinctive histological pattern associated with dedifferentiated liposarcoma. Histopathology 1998;33: 414-424.

22 Johnson MR, Seys AR, Roth CW, et al. Assessment of carboxypeptidase M (CPM) amplification: a surrogate marker for MDM2 amplification in the discrimination of well-differentiated liposarcomas from lipomas. ASHG Meeting Booklet. Phildelphia. Abstract 1190. 2008, p.246.

23 Deiteren K, Hendriks D, Scharpé S, et al. Carboxypeptidase M: multiple alliances and unknown partners. Clinica Chimica Acta 2009;399:24-39. 